\title{
Practical accuracy of VRS RTK outside the Malaysian Real Time Kinematic Network (MyRTKnet)
}

\begin{abstract}
Real Time Kinematic become a popular high precision technique in Malaysia. However, limitation of the distance for base station to rover station, this technique still difficult to implement. Virtual Reference Station (VRS) is a new concept developed by Trimble Terrasat is efficient method to overcome the limitation of classical RTK. The Malaysian Real-Time Kinematic Network (MyRTKnet) has been developed to facilitate RTK positioning in Malaysia. MyRTKnet consists 78 reference stations located at Peninsular Malaysia, Sabah and Sarawak. The most Northern reference station in Peninsular Malaysia located at Arau, Perlis. The gap distance from the Arau station to the Malaysia - Thailand border is around 35 $\mathrm{km}$. This paper will discuss the practical accuracy of the RTK positioning using VRS generated by MyRTKnet outside the network. In this study, four points outside the MyRTKnet network were observed continuously in VRS RTK mode. Distances between rover station and the nearest physical reference station are $1 \mathrm{~km}, 10 \mathrm{~km}, 20 \mathrm{~km}$ and $30 \mathrm{~km}$. It was found that the VRS generated using MyRTKnet can be achieved up to $6 \mathrm{~cm}$ in horizontal component and up to $8 \mathrm{~m}$ in vertical component even the positions of the rover stations are outside the MyRTKnet.
\end{abstract}

